\title{
Enhanced Fatigue Property of Welded S355J2W Steel by Forming a Gradient Nanostructured Surface Layer
}

\author{
Lu An ${ }^{1} \cdot$ Yan-Tao Sun ${ }^{1} \cdot$ Shan-Ping Lu ${ }^{1} \cdot$ Zhen-Bo Wang $^{1}$ \\ Received: 1 November 2019 / Revised: 23 December 2019 / Published online: 21 April 2020 \\ (c) The Chinese Society for Metals (CSM) and Springer-Verlag GmbH Germany, part of Springer Nature 2020
}

\begin{abstract}
Welded joints are usually characterized by microstructural and compositional inhomogeneities, which may significantly degrade their fatigue properties and result in unpredictable failures. The present work demonstrates a novel and simple method to effectively optimize the microstructure in the surface layer and promote the fatigue properties of welded specimens. By a recently developed approach—surface mechanical rolling treatment (SMRT), a gradient nanostructured surface layer is formed on welded $\mathrm{S} 355 \mathrm{~J} 2 \mathrm{~W}$ steel specimens. The mean grain size is refined to nanometer scale, and the hardness is significantly enhanced in the SMRT surface layer. Independent of the initially inhomogeneous microstructure and hardness distributions, the microstructure and hardness distributions in the surface layers are comparable on different zones of a welded specimen after SMRT with the same procedure. Consequently, fatigue property of the SMRT specimens is significantly enhanced relative to that of the as-welded specimens within the high cycle fatigue regime.
\end{abstract}

Keywords Gradient nanostructured $\cdot$ Surface mechanical rolling treatment $\cdot$ S355J2W steel $\cdot$ Welded $\cdot$ Fatigue

\section{Introduction}

Welding technologies are widely applied to joint metallic components in industries. As we know, welded joints are usually characterized by having microstructural and compositional inhomogeneities, such as segregation, precipitation, coarse grains and even porosities [1,2]. These defects significantly degrade the fatigue properties of welded components $[3,4]$, which may result in unpredictable failures. For instance, several train accidents have occurred due to fatigue fracture of the bogie frames, which were welded and operated under cyclic dynamic loading during service $[5,6]$. In order to improve fatigue properties of welded joints, various post-welding techniques have been developed, including arc re-melting [7], heat treatment [8], surface polishing and plastic deformation $[3,9]$.

Available online at https://link.springer.com/journal/40195.

Zhen-Bo Wang

zbwang@imr.ac.cn

1 Shenyang National Laboratory for Materials Science, Institute of Metal Research, Chinese Academy of Sciences, Shenyang 110016, China
Fatigue properties can be significantly enhanced in metals by forming a gradient nanostructured (GNS) surface layer, in which the mean grain size is in the nanometer scale at the top surface and increases gradually to the micrometer scale in the sample interior. This has been observed by different works in Ni-based superalloy [10], 316L stainless steel [11, 12], bearing steel [13] and Cr-Mo steel [14]. For example, in GNS 316L stainless steel, the fatigue limit (i.e., the strength at a life $>2 \times 10^{6}$ cycles) was increased by $\sim 78 \%$ under the stress-controlled fatigue mode, and the fatigue life was increased $\sim 30$ times under the strain-controlled mode (with the total strain amplitude of $0.3 \%$ ), in comparison with those of the coarse-grained counterparts [12]. Further analysis demonstrated that the gradient nanostructures provided a more effective approach to enhance the fatigue properties than homogenous nanostructures did. The initiation and propagation of fatigue cracks were remarkably impeded by the strong nanostructured surface layer and the ductile coarse-grained interior, respectively. Therefore, the formation of a GNS surface layer on welded specimens is expected to significantly enhance their fatigue properties.

As a kind of high-strength low-alloy steel, S355J2W steel is welded to design bogie frames of high-speed trains reaching speeds $>300 \mathrm{~km} \mathrm{~h}^{-1}$. Higher fatigue and mechanical properties are therefore crucial in the welded joints in 
order to maintain effective and long-term performance. In the present work, the welded S355J2W steel specimens were submitted to a recently developed technique named surface mechanical rolling treatment (SMRT), which has been successfully applied to produce a thicker GNS surface layer with smooth surface on various steels in the previous works $[11,15]$. Subsequently, the microstructure and hardness distribution were studied in different regions of the SMRT specimens. And the effects of the GNS surface layer on the fatigue property of the welded specimens were compared with those of the as-welded specimens.

\section{Experimental}

The base metal (BM) used in this work was commercial hot-rolled S355J2W steel. Two plates with a thickness of $\sim 12 \mathrm{~mm}$ were welded by the metal active gas (MAG) welding technology using a low-carbon welding wire, as described in the previous work [1]. The chemical compositions of the BM and the welding wire are given in Table 1, and the welded sample is schematically illustrated in Fig. 1a. The geometry of the joint was $\mathrm{Y}$ groove of $60^{\circ}$. A fourlayer welding procedure was employed under the shielding gas of $82 \% \mathrm{Ar}+18 \% \mathrm{CO}_{2}$. Fatigue samples with a dog-bone shape as shown in Fig. $1 \mathrm{~b}$ were prepared from the plate by electro-spark discharge cutting, for SMRT processing and for as-welded counterparts.

SMRT was performed on a lathe at room temperature as described in the previous works $[11,16]$. As schematically illustrated in Fig. 1c, a WC-Co cermet ball settled on a tool tip was pressed into the sample surface (with a preset depth $a_{\mathrm{p}}$ of $20 \mu \mathrm{m}$ ), while the sample rotated around the axis at a velocity $\left(v_{1}\right)$ of 600 turns per min. Meanwhile, the ball moved along the axis from one shoulder to the other shoulder of the fatigue sample (across the BM, HAZ and WM sections as shown in Fig. 1 b) at a velocity $\left(v_{2}\right)$ of $0.02 \mathrm{~mm}$ per turn. The ball was rotatable and lubricated by cycling oil. In the present work, the procedure mentioned above was repeated 4 times, with constant $v_{1}$ and $v_{2}$, and stepwise increased $a_{\mathrm{p}}$. As the results, the surface layer of the treated sample was deformed multiple times under high strain rates and grains were expected to be gradually refined into nanosize.

Microstructures of different sections in the welded samples before and after SMRT were observed by using an FEI Nova NanoSEM 430 scanning electronic microscope (SEM)

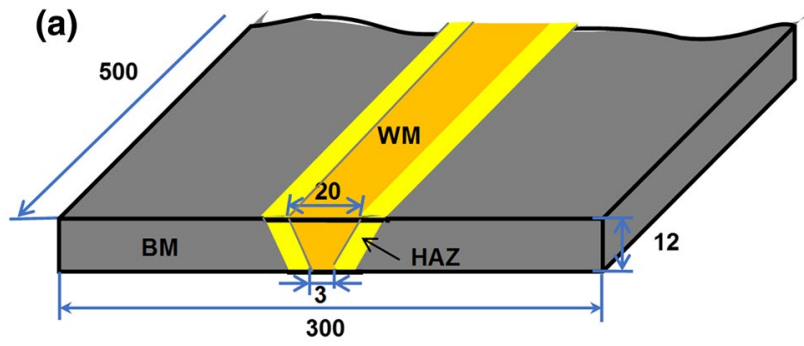

(b)
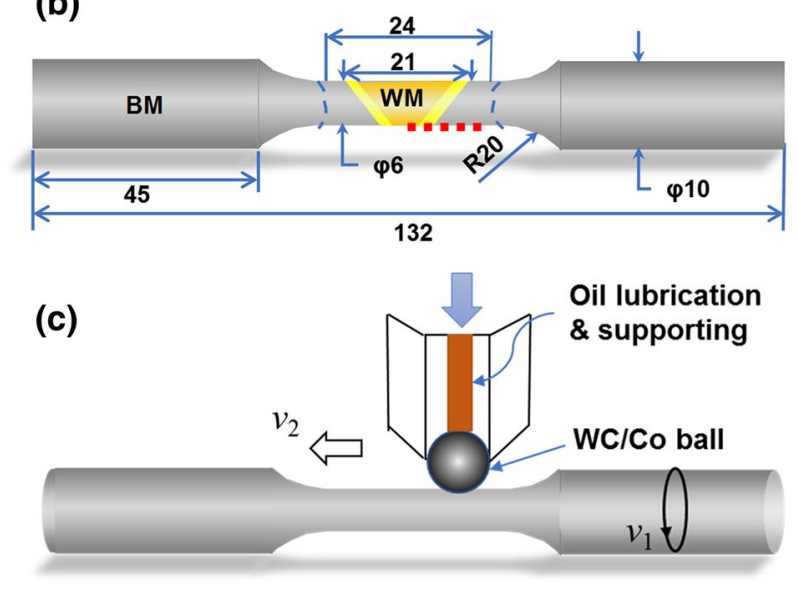

Fig. 1 Schematic illustrations of a a welded plate of S355J2W steel, b a cylinder dog-bone-shaped fatigue sample cut from a, $\mathbf{c}$ the SMRT process. The red dot line on $\mathbf{b}$ marks the position for surface hardness measurements in Fig. 6. WM and HAZ denote weld metal and heataffected zone, respectively (unit: $\mathrm{mm}$ )

operated at a voltage of $15 \mathrm{kV}$. Furthermore, detailed microstructural characterizations were observed by using a JEOL2010 transmission electron microscope (TEM) operated at a voltage of $200 \mathrm{kV}$. Thin foils for TEM observations of the top surface layers on different sections of the SMRT sample were cut by electro-spark discharge technique, mechanically polished, dimpled and finally ion milled from the matrix side. Residual stresses in the SMRT surface layer were measured by X-ray diffraction with a Co $K_{\alpha}$ radiation on a Bruker D8 Discover unit, using the classical $2 \theta-\sin ^{2} \psi$ method and the (211) Bragg diffraction peak of ferrite. And surface morphologies of the samples were monitored by using an Olympus LEXT OLS4000 confocal laser scanning microscope (CLSM).

Microhardness variations along the depth from the treated surface were measured on cross-sectional samples corresponding to different regions sectioned from a SMRT
Table 1 Chemical compositions of the $\mathrm{BM}$ and welding wire $(\mathrm{wt} \%)$

\begin{tabular}{llllllllllll}
\hline Material & $\mathrm{C}$ & $\mathrm{Si}$ & $\mathrm{Mn}$ & $\mathrm{Ni}$ & $\mathrm{Cu}$ & $\mathrm{Cr}$ & $\mathrm{P}$ & $\mathrm{S}$ & $\mathrm{Ti}$ & $\mathrm{Nb}$ & $\mathrm{Fe}$ \\
\hline $\mathrm{BM}$ & 0.075 & 0.29 & 1.26 & 0.2 & 0.3 & 0.45 & 0.007 & 0.007 & 0.014 & 0.016 & $\mathrm{Bal}$ \\
Welding wire & 0.044 & 0.43 & 1.07 & 0.89 & 0.43 & 0.016 & 0.006 & 0.004 & 0.052 & 0.01 & $\mathrm{Bal}$ \\
\hline
\end{tabular}


sample. In addition, the distribution of surface hardness along the distance from the center of welded joint was measured on both the SMRT and as-welded samples from a planar view along the sample axis, as marked in Fig. 1. Measurements were taken with a maximum load of $10 \mathrm{~g}$ and a holding duration of $5 \mathrm{~s}$, by using a Qness Q10 A + microhardness tester fitted with a Vickers indenter. In addition, tensile properties of the SMRT and as-welded samples were measured by using a Shimadzu AG-Xplus testing machine with a strain rate of $5 \times 10^{-4} \mathrm{~s}^{-1}$.

Axial tension-compression fatigue tests were performed by using an Instron 8874 electro-hydraulic tester at room temperature. The stress control fatigue properties were studied according to the GB/T 3075 Method of axial fatigue testing of metals, with a zero-mean stress $(R=-1)$ and a frequency of $10 \mathrm{~Hz}$. A fatigue test was stopped when the sample failed or survived by $2 \times 10^{6}$ cycles. The corresponding maximum stress was taken as the fatigue strength. Fracture surfaces of fatigued samples were observed by SEM on an FEI Nova NanoSEM 430 unit.

\section{Results}

\subsection{Microstructure of Samples Before and After SMRT}

Different microstructures are observed in different regions of the welded plate, as shown in Fig. 2. In the BM region, a typical banded structure of ferrite and pearlite is formed after hot rolling. With decreasing distance to the welded joint, the banded structure gradually transforms into a normalized or over-heated state in the HAZ due to the heating effects from the molten metal during welding. In the WM region, the microstructure is characterized by ferrite grains with some cementite precipitates along grain boundaries. Further microstructural observations show that the welded joint is composed of a columnar grain zone and a fine grain zone, mostly due to the applied multi-pass MAG welding process. With the addition of a small amount of Ti, the sizes of ferrite grains in both zones are significantly refined and more acicular ferrite is formed in the columnar grain zone. Such optimized microstructures result in a higher impact property in the Ti-bearing WM compared to the WM without Ti addition [1].

In the surface layer of the SMRT sample, clear evidence of severe plastic deformation and microstructure refinement can be observed in all the regions of BM, HAZ and WM, as shown by cross-sectional morphologies in Fig. 3a-c, respectively. The degrees of plastic deformation and microstructure refinement gradually increase with decreasing depth to the treated surface, and the microstructure is difficult to identify by SEM in the top surface layer of $\sim 50 \mu \mathrm{m}$ in thickness. TEM observations confirm that nanosized grains are formed in the top surface layer, as shown in Fig. 4. It is noticed that slight differences exist in the mean grain sizes of different regions. Statistic measurements show that the mean grain sizes of ferrite are about 50,70 and $30 \mathrm{~nm}$ in the top surface layers of the BM, HAZ and WM regions, respectively.

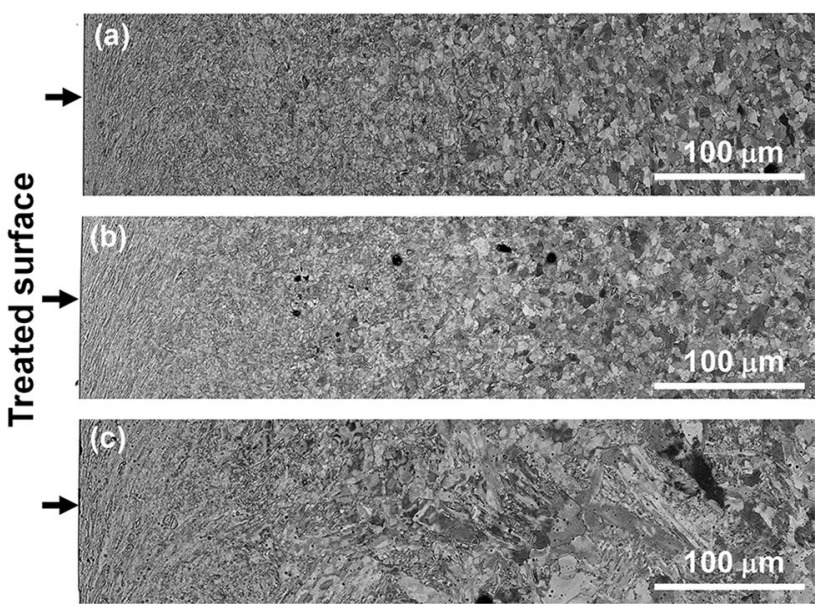

Fig. 3 Cross-sectional SEM morphologies of different regions in the SMRT sample: a BM, b HAZ, c WM
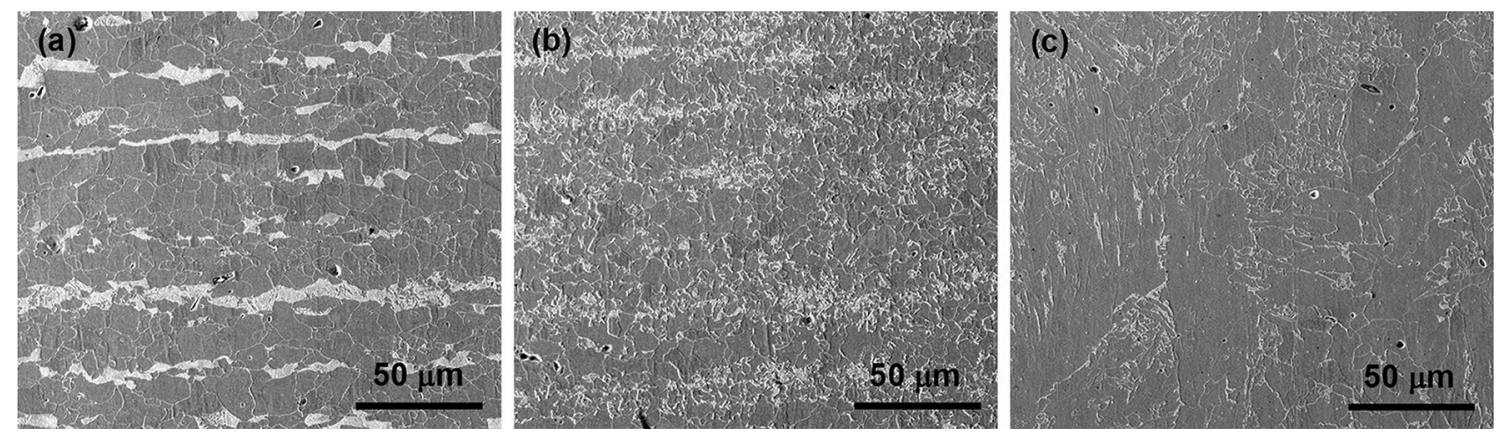

Fig. 2 Typical SEM morphologies of different regions (as indicated in Fig. 1) in the as-welded S355J2W steel plate: a BM, b HAZ, c WM 

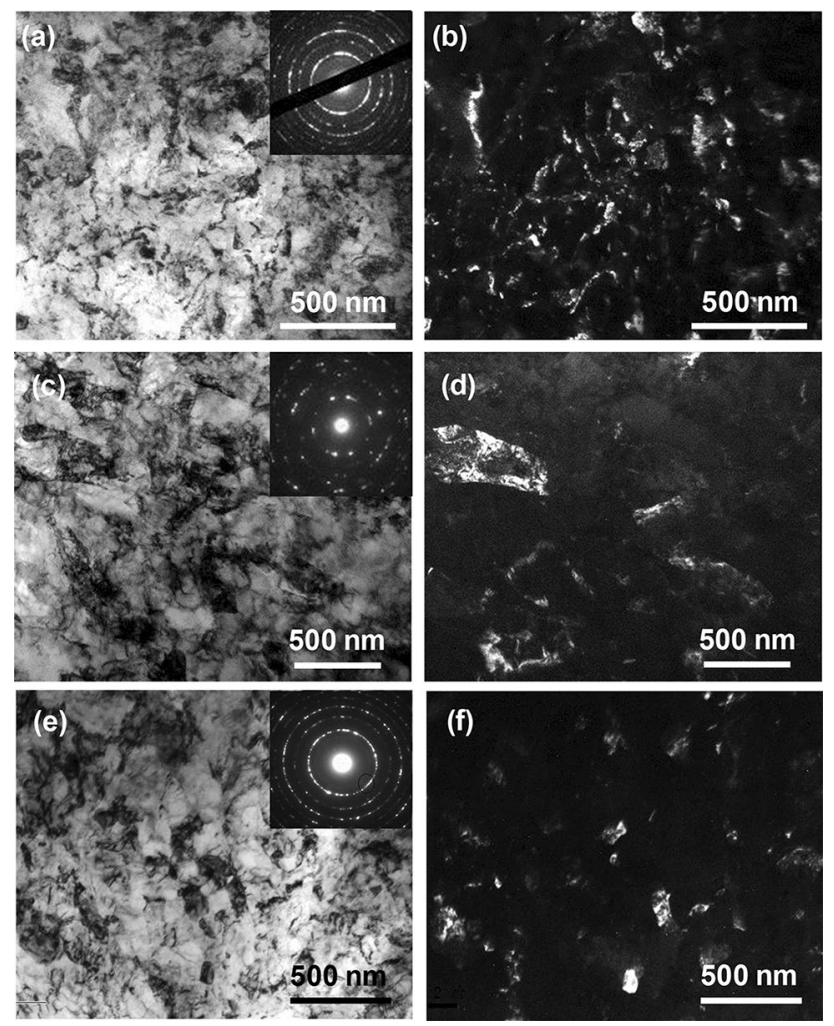

Fig. 4 Typical TEM images of the topmost surface layers of different regions in the SMRT sample: a, b BM, c, d HAZ, e, f WM. a, c, e are bright-field TEM images with corresponding SAED patterns inserted. $\mathbf{b}, \mathbf{d}, \mathbf{f}$ are dark-field TEM images taken from the (110) diffraction of ferrite (the first ring of the SAED pattern) in $\mathbf{a}, \mathbf{c}, \mathbf{e}$, respectively

CLSM observations show that the SMRT surface is quite smooth, with a surface roughness $\left(R_{\mathrm{a}}\right)$ of $\sim 0.13 \mu \mathrm{m}$. In comparison, the surface roughness of the as-welded samples for fatigue tests is controlled to be $\sim 0.18 \mu \mathrm{m}$ after mechanical polishing.

\subsection{Mechanical Properties}

A similar gradient distribution of microhardness along the depth has been achieved in the surface layers of different regions in the SMRT samples, as shown in Fig. 5. The hardness typically reaches $\sim 3.1 \mathrm{GPa}$ at the depth of $\sim 10 \mu \mathrm{m}$ and gradually decreases to respective matrix values at the depth of $\sim 600 \mu \mathrm{m}$. It is noted that the first data point measured on a cross-sectional sample is $\sim 10 \mu \mathrm{m}$ in depth from the treated surface, because the derived value may be inaccurate (usually smaller) due to the interference between the plastic deformation zone around the indent and the sample edge at a smaller depth. To check the situation in the top surface layer, the hardness is measured on the treated surface of the SMRT sample from a planar view. As shown in Fig. 6, the surface hardness reaches $\sim 3.5 \mathrm{GPa}$ and keeps almost constant at different regions of the welded joint. In comparison, the surface

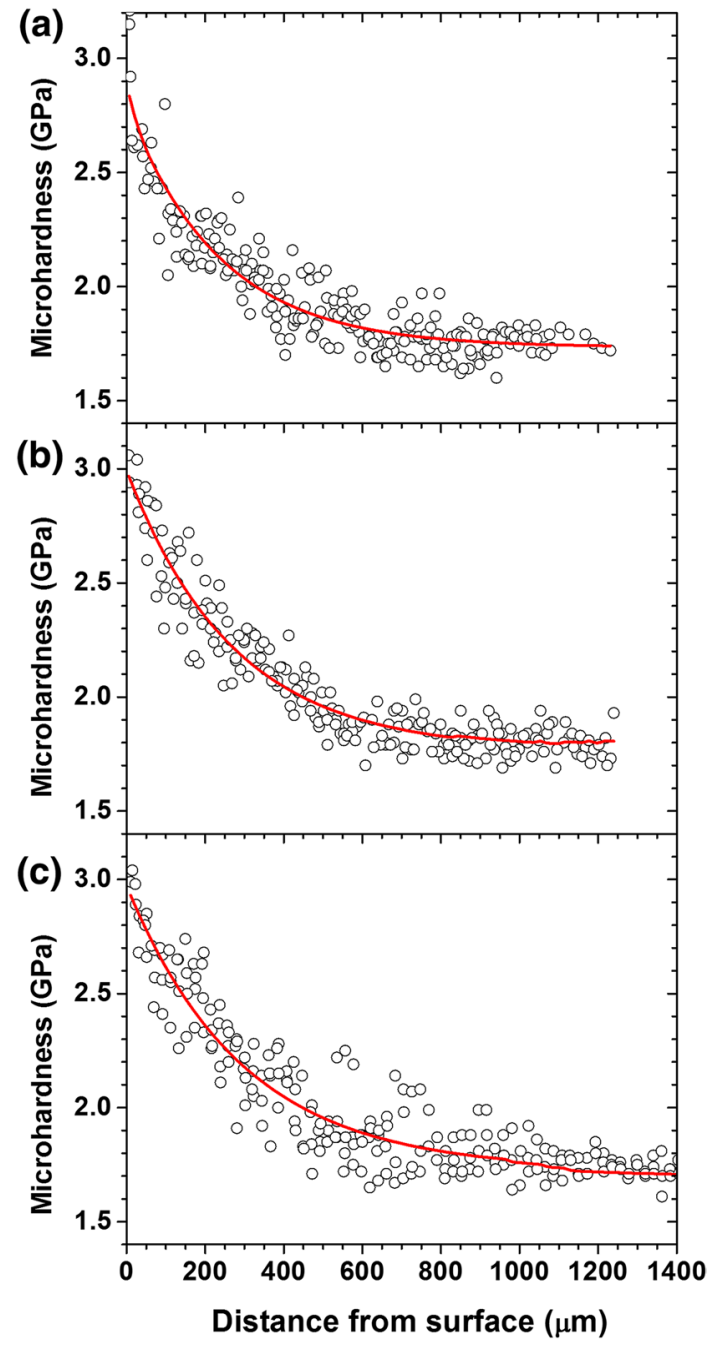

Fig. 5 Variations of microhardness with the distance from the treated surface for different regions in the SMRT sample: a BM, b HAZ, c WM. Measurements were taken on the surface layer from a cross-sectional view. Each point denotes an independent measurement

hardness varies with distance from the welded-joint center on the as-welded sample, with a maximum value $(\sim 2.0 \mathrm{GPa})$ at the $\mathrm{HAZ}$ region and $\sim 1.7 \mathrm{GPa}$ at the $\mathrm{WM}$ and $\mathrm{BM}$ regions.

The strength of the welded samples is enhanced after SMRT, mostly due to the increased hardness/strength in the surface layer. Tensile tests show that the ultimate strength increases from $\sim 522 \mathrm{MPa}$ of the as-welded samples to $\sim 542 \mathrm{MPa}$ of the SMRT samples.

\subsection{Fatigue Properties}

The fatigue properties of the SMRT samples are compared with those of the as-welded samples in Fig. 7. While the fatigue lives of both samples are comparable at a stress amplitude of $\sim 340 \mathrm{MPa}$, the fatigue lives of the SMRT samples are higher than those of the as-welded samples at lower 


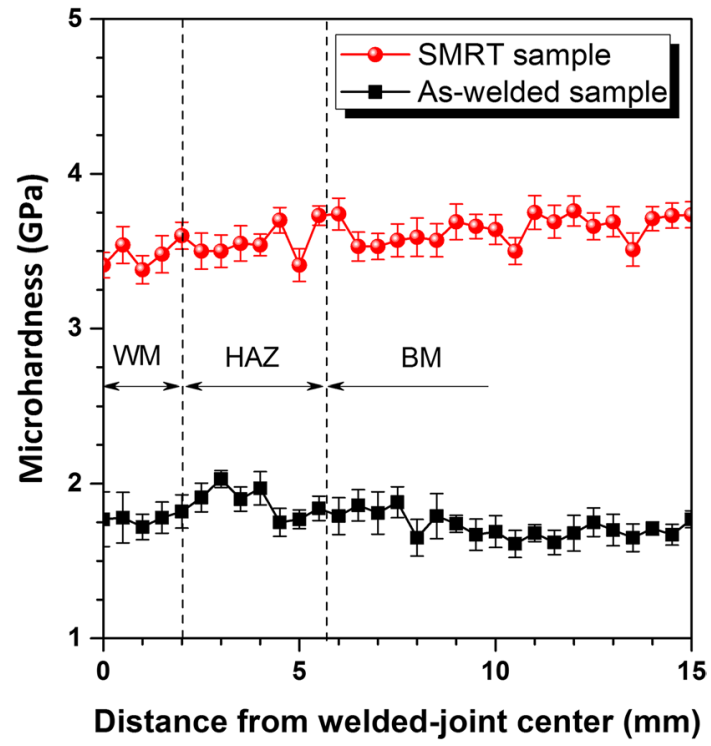

Fig. 6 Distributions of surface hardness along the distance from the welded-joint center on the SMRT and as-welded samples. Measurements were taken on the sample surface from a planar view, along the red dot line marked in Fig. 1b. Each point was averaged from at least 3 measurements

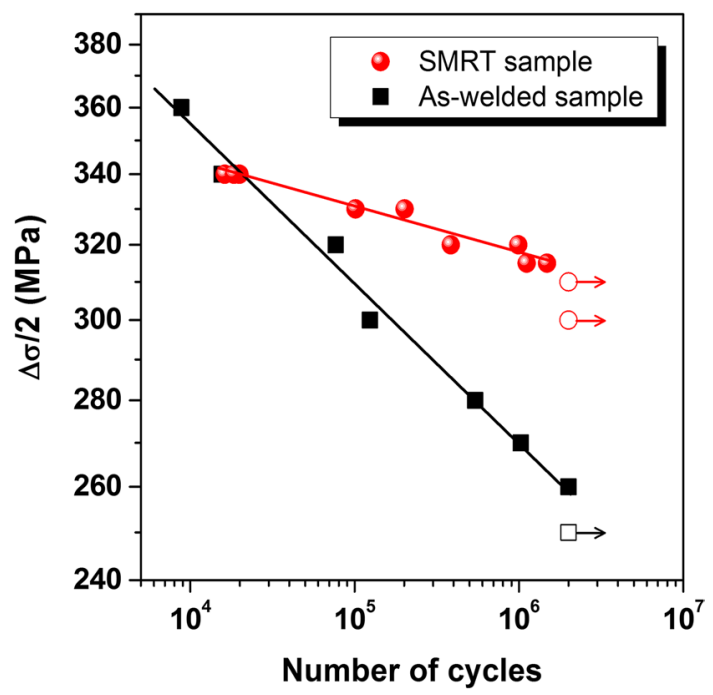

Fig. $7 S-N$ curves of the SMRT and as-welded samples. Open symbols (with arrows) indicate tests terminated without fracture after $2 \times 10^{6}$ cycles

stress amplitudes (i.e., within the high cycle fatigue regime), and the improvement increases with decreasing stress amplitude. For example, the fatigue life of the SMRT samples reaches $\sim 1.3 \times 10^{6}$ cycles at $\sim 315 \mathrm{MPa}$ and exceeds $2 \times 10^{6}$ cycles at $310 \mathrm{MPa}$. In comparison, the fatigue life of the as-welded samples is only $1.2 \times 10^{5}$ cycles at $300 \mathrm{MPa}$. The fatigue limit increases from $\sim 250 \mathrm{MPa}$ of the as-welded samples to $310 \mathrm{MPa}$ of the SMRT samples.
Observations of fractured samples show that the initiation of fatigue cracks typically occurs at the sample surface under higher stress amplitudes in both the SMRT and as-welded samples, while it may occur at the subsurface (or interior) under stress amplitudes close to the fatigue limits, as shown in Fig. 8. Detailed analysis of microstructure and chemical composition reveals that the sites of internal crack initiation are characterized by defects such as oxide inclusions, chemical inhomogeneities and micro-cracks. In addition, different from the fact that all the fracture positions are within the HAZ or WM zones in the as-welded samples, several SMRT samples are found to fracture within the BM zone.

\section{Discussion}

\subsection{Formation of the GNS Surface Layer}

TEM observations (Fig. 4) indicate that grain sizes have been refined into a nanoscale in the top surface layers on different regions of the SMRT samples. Meanwhile, the corresponding gradient distributions in the microstructure (Fig. 3) and microhardness (Fig. 5) along the depth of the welded specimen suggest gradient distributions of grain sizes in the SMRT surface layers. These confirm that the GNS surface layer has been fabricated on different regions of the welded specimen by SMRT. As revealed in the previous works [17-19], the formation of GNS surface layers in low-alloy steels or carbon steels is typically dominated by dislocation activities under surface gradient deformation processes. In the deeper region of the surface layer, dislocation walls and dislocation tangles are formed in the original ferrite grains under accumulative strains, and then, they are gradually transformed into sub-boundaries with small misorientations and decreased spaces due to more intensive interactions with dislocations under increasing strains and strain rates in the sub-surface layer. Finally, the grain boundaries with high misorientations and grains with nanoscale sizes are formed in the top surface layer under high strains and strain rates. In addition, higher stress concentrations may be achieved at interfaces between the ferrite matrix and the cementite particles (or second phases) due to the inhomogeneous plasticity in the SMRT surface layer. When the local stress concentration exceeds the shear strength of cementite, plastic strain may be achieved in the particles, leading to their deformation and fragmentation or even decomposition [15, $16,20]$. This is the reason why a similar SEM morphology is observed in the SMRT surface layers initially with different cementite distributions on the BM, HAZ and WM zones (see Fig. 3), and no cementite can be detected by TEM and SAED in the surface layers after SMRT (see Fig. 4).

However, it is noticed that different grain sizes are obtained in the top surface layers on different zones of the 

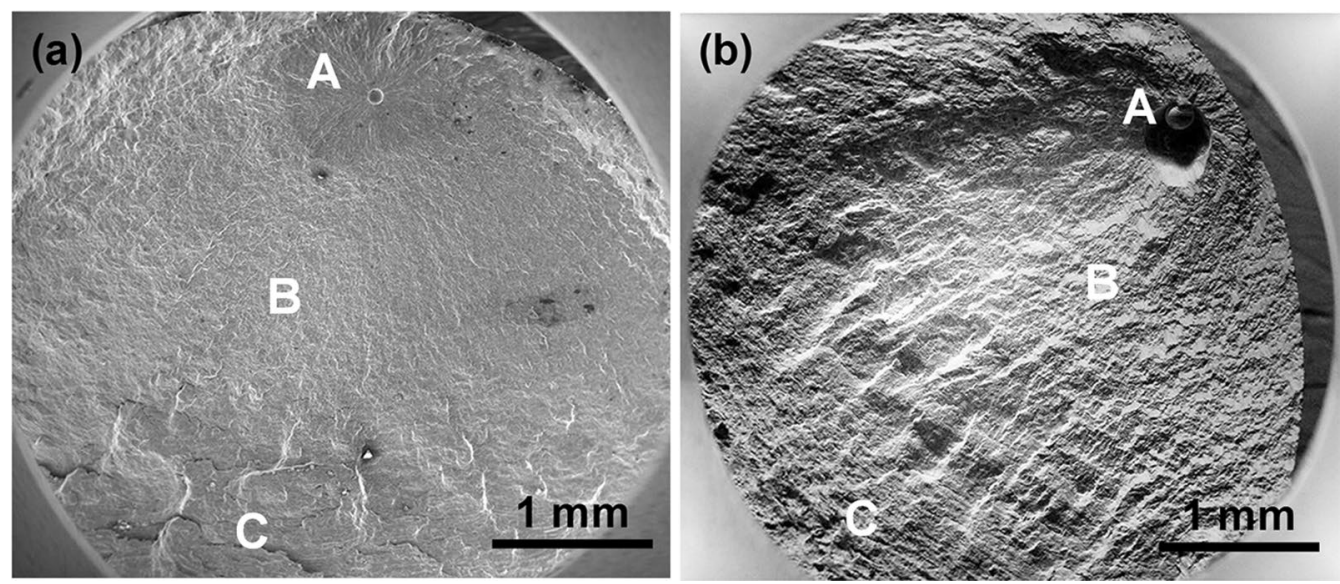

Fig. 8 SEM morphologies of fracture surface of a the SMRT sample fatigued at $320 \mathrm{MPa}$, b the as-welded sample fatigued at $280 \mathrm{MPa}$. The regions of crack initiation, crack propagation, and instant rupture are marked by $\mathrm{A}, \mathrm{B}$, and $\mathrm{C}$, respectively

MAG-welded specimen by TEM observations, while the same SMRT parameters are applied. This is related to the different initial microstructures and mechanical properties in these zones. For example, the mean grain size is the largest $(\sim 70 \mathrm{~nm})$ in the top surface layer on the HAZ, in which the initial hardness is the highest and the microstructure refinement dominated by dislocation activities is expected to be slightly more difficult than in other zones. Subsequently, surface layers with homogeneous mechanical properties (i.e., the similar surface hardness and hardness-depth distribution) are obtained on different zones of the welded specimen after SMRT, although they initially possess a different microstructure and hardness.

\subsection{Fatigue Mechanisms}

The effect of surface roughness on the fatigue property can be neglected, while surfaces of both the SMRT and as-welded samples were controlled to similar conditions $\left(R_{\mathrm{a}}<0.2 \mu \mathrm{m}\right)$. Therefore, the enhancement in fatigue property is expected to be mostly related to the formation of GNS surface layers with higher hardness and microstructural homogeneity in the SMRT samples. Conventionally, surface extrusions and intrusions develop on coarse-grained samples due to the formation of persistent slip bands and other localized dislocation structures under cyclic loading during fatigue tests $[11,21]$. And a fatigue failure usually initiates at the sample surface due to the stress concentration or higher initial stress intensity factor range $(\Delta K)$ around defects [22]. On the GNS samples, the development of extrusions and intrusions will be impeded because of the higher hardness and strength in the surface layer [13]. In addition, defects such as oxide inclusions and chemical inhomogeneities in the surface layer of the as-welded samples will be significantly refined during SMRT. Therefore, the stress concentration or $\Delta K$ value will be decreased in the GNS samples, and a higher fatigue stress is expected to initiate the fatigue failure. Furthermore, while the microstructural and chemical inhomogeneities mostly exist in the HAZ and WM regions of the welded joint, a fatigue fracture is expected to initiate from these two regions in the as-welded samples. With the significant refinement of these defects in the surface layer, the fatigue fracture possibly initiates from the $\mathrm{BM}$ region or the subsurface layer (around a defect) in the GNS samples.

It is to be noted that distinct surface compressive residual stress can be introduced into the GNS surface layer by the gradient distribution of strains during SMRT $[11,16]$. In the present work, the compressive residual stresses reach $702 \pm 39$ and $333 \pm 33 \mathrm{MPa}$ along the axial and the transverse directions, respectively, in the SMRT surface layer. This will modify the complex residual stresses (sometimes in tensile or tensile-compressive transitional states) in the welded metals [23, 24]. In addition to the contributions from higher hardness and microstructural homogeneity due to microstructural refinement in the GNS surface layer, the fatigue property is expected to be further enhanced, while the surface compressive residual stress decreases the mean stress, which also retards the initiation and propagation of cracks [22, 23, 25].

With the increase in stress amplitude, compressive residual stress will relax significantly in the GNS surface layer at the earlier stage of fatigue tests [12,13]. Meanwhile, cyclic hardening may occur in the interior of the SMRT samples, similar to that in the as-welded samples. Therefore, the enhancement of the fatigue property is expected to become smaller in the SMRT samples relative to in the as-welded samples. For example, in the previous work [12], the matrix hardness was found to increase remarkably and the gradient distribution of hardness along the depth of the welded 
specimen became insignificant in a GNS bearing steel sample fatigued under a stress amplitude much higher than the fatigue limit (while the matrix hardness kept unchanged in the GNS samples fatigued under a stress close to the fatigue limit), resulting in comparable fatigue properties in the GNS and as-welded samples.

\section{Conclusion}

GNS surface layers were formed on different zones of a welded specimen of S355J2W steel by SMRT in this study. The mean grain sizes were about 50,70 and $30 \mathrm{~nm}$ at the top surface on the BM, HAZ and WM zones, respectively, and gradually increased with increasing depth. As a result, the hardness was remarkably enhanced on the treated surface and gradually decreased with the depth. It was noticed that the surface hardness was observed to remain constant $(\sim 3.5 \mathrm{GPa})$, while the thickness of the hardened (microstructure-refined) surface layers was $\sim 600 \mu \mathrm{m}$ on different zones of the welded specimen after SMRT with the same procedure, which was independent of the initial inhomogeneous distributions of microstructure and hardness. Fatigue property of the SMRT samples was significantly enhanced within the high cycle fatigue regime under tension-compression tests, with a fatigue limit of $\sim 310 \mathrm{MPa}$ compared to $\sim 250 \mathrm{MPa}$ in the as-welded samples. The higher hardness, microstructural homogeneity and compressive residual stress in the GNS surface layer were discussed to mainly contribute to the enhancement in fatigue property. The present work demonstrated a novel and simple surface modification approach to effectively promote the fatigue properties of welded joints of structural components.

Acknowledgements This work was financially supported by the National Key Research and Development Program of China (Nos. 2017YFA0204401 and 2017YFA0204403), the Liaoning Revitalization Talents Program (No. XLYC1808008) and the Shenyang National
Laboratory for Materials Science. The authors are grateful to Prof. K. Lu from Shenyang National Laboratory for Materials Science for constructive discussion on this work, and to Dr. W.C. Dong for providing welded S355J2W steel plates.

\section{References}

[1] S.P. Lu, X. Wang, W.C. Dong, Y.Y. Li, ISIJ Int. 53, 96 (2013)

[2] H. Kokawa, M. Shimada, M. Michluchi, Z.J. Wang, Y.S. Sato, Acta Mater. 55, 5401 (2007)

[3] D. Li, H.N. Chen, H. Xu, Surf. Eng. 25, 15 (2009)

[4] H. Atwa, N.M. Mawsouf, M.Y.A. Younan, Eng. Fract. Mech. 44, 921 (1993)

[5] B.L. He, Y.X. Yu, H.H. Yu, J.P. Shi, Y.F. Zhu, Curr. Nanosci. 8, 17 (2012)

[6] J.S. Kim, Eng. Fail. Anal. 13, 1326 (2006)

[7] T. Dahle, Int. J. Fatigue 20, 677 (1998)

[8] S. Malarvizhi, V. Balasubramanian, Mater. Des. 32, 1205 (2011)

[9] S.H. Han, J.W. Han, Y.Y. Nam, I.H. Cho, Fatigue Fract. Eng. Mater. Struct. 32, 573 (2009)

[10] L.L. Shaw, J.-W. Tian, A.L. Ortiz, K. Dai, J.C. Villegas, P.K. Liaw, R. Ren, D.L. Klarstrom, Mater. Sci. Eng. A 527, 986 (2010)

[11] H.W. Huang, Z.B. Wang, J. Lu, K. Lu, Acta Mater. 87, 150 (2015)

[12] Y.B. Lei, Z.B. Wang, J.L. Xu, K. Lu, Acta Mater. 168, 133 (2019)

[13] K. Zhang, Z.B. Wang, K. Lu, Mater. Res. Lett. 5, 258 (2017)

[14] M.S. Suh, C.M. Suh, Y.S. Pyun, Fatigue Fract. Eng. Mater. Struct. 36, 769 (2013)

[15] K. Zhang, Z.B. Wang, J. Mater. Sci. Technol. 34, 1676 (2018)

[16] H.W. Huang, Z.B. Wang, X.P. Yong, K. Lu, Mater. Sci. Technol. 29, 1200 (2013)

[17] N.R. Tao, Z.B. Wang, W.P. Tong, M.L. Sui, J. Lu, K. Lu, Acta Mater. 50, 4603 (2002)

[18] S.D. Lu, Z.B. Wang, K. Lu, J. Mater. Sci. Technol. 26, 258 (2010)

[19] L.C. Fu, D.Y. Li, Adv. Eng. Mater. 15, 476 (2013)

[20] L. Zhou, G. Liu, X.L. Ma, K. Lu, Acta Mater. 56, 78 (2008)

[21] H. Mughrabi, Int. J. Fatigue 28, 1501 (2006)

[22] K. Shiozawa, M. Murai, Y. Shimatani, T. Yoshimoto, Int. J. Fatigue 32, 541 (2010)

[23] Z.Y. Feng, X.J. Di, S.P. Wu, D.P. Wang, X.Q. Liu, Acta Metall. Sin. (Engl. Lett.) 31, 541 (2018)

[24] L. Li, Z. Wan, Z. Wang, C. Ji, Acta Metall. Sin. (Engl. Lett.) 22, $202(2009)$

[25] M.K. Khan, M.E. Fitzpatrick, Q.Y. Wang, Y.S. Pyoun, A. Amanov, Fatigue Fract. Eng. Mater. Struct. 41, 844 (2018) 